\title{
Anreicherung der leichten Zinkisotope durch elektrolytische Überführung in geschmolzenem Zinkchlorid
}

\author{
Von Alfred Klemm, Heinrich Hintenberger* und Arnold Lundén \\ Aus dem Institut für Physik, Chalmers Technische Hochschule, Göteborg, \\ und dem Max-Planck-Institut für Chemie, Mainz \\ (Z. Naturforschg. 6 a, 489—494 [1951]; eingegangen am 2. Juli 1951)
}

\begin{abstract}
An der Grenze zwischen $\mathrm{PbCl}_{2}$ und $\mathrm{ZnCl}_{2}$ wurde durch elektrolytische Überführung (Dauer 3 Tage, Stromstärke $230 \mathrm{~mA}$, Stromdichte $4,27 \mathrm{~A} / \mathrm{cm}^{2}$, Verschiebung des Chlors relativ zum Zink 3,4 m, Vervielfachung 167, praktische Reichweite der Anreicherung $4 \mathrm{~cm}$, Temperatur im Trennrohr $650^{\circ} \mathrm{C}$ ) die Isotopenverteilung beim $\mathrm{Zn}$ zugunsten der leichten Isotope verschoben. Zwischen der besten Fraktion (59 mg Zink) und natürlichem Gemisch war für das Isotopenpaar

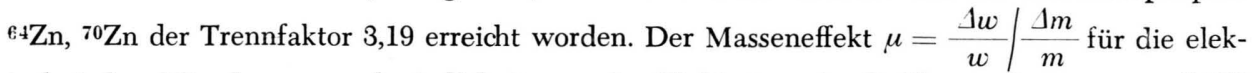
trolytischen Wanderungsgeschwindigkeiten $w$ der Zinkisotope im $\mathrm{ZnCl}_{2}$ wurde $\mathrm{zu} \mu=-0,078$ bestimmt.
\end{abstract}

$\mathrm{D}$ ie Kette - $/$ Kohle $/ \mathrm{Pb} / \mathrm{PbCl}_{2} / \mathrm{ZnCl}_{2} / \mathrm{Cl}_{2}$ Kohle $/+$ wurde 3 Tage lang mit $230 \mathrm{~mA}$ elektrolysiert, wobei sich die Grenze zwischen der $\mathrm{PbCl}_{2}$ - und $\mathrm{ZnCl}_{2}-$ Schmelze in einem Diaphragma mit $0,054 \mathrm{~cm}^{2}$ freiem Querschnitt befand, so daß dort eine Stromdichte von $4,27 \mathrm{~A} / \mathrm{cm}^{2}$ herrschte. Dadurch wurden bei einer Dichte des $\mathrm{ZnCl}_{2}\left(650^{\circ} \mathrm{C}\right)$ von $2,3 \mathrm{~g} / \mathrm{cm}^{3}$ die Zinkionen relativ zu den Chlorionen um $340 \mathrm{~cm}$ verschoben. In Analogie zu früheren Versuchen ${ }^{1}$ war an der Grenze $\mathrm{PbCl}_{2} / \mathrm{ZnCl}_{2}$ eine Anreicherung der leichten Zinkisotope zu erwarten. Für eine Anreicherung der schweren Bleiisotope an dieser Grenze war das Diaphragma auf der Seite des Bleichlorids zu kurz.

Vorversuche hatten gezeigt, daß während der Elektrolyse im mit $\mathrm{ZnCl}_{2}$ gefüllten Teil des Diaphragmas gasgefüllte Hohlräume entstehen können, die zum Abwandern der Grenze $\mathrm{ZnCl}_{2}-\mathrm{PbCl}_{2}$ nach der Kathode hin und zur Stromunterbrechung führen. Durch sorgfältiges Fernhalten von Wasser ließ sich diese Störung vermeiden. Das Zinkchlorid wurde deshalb in einem Seitenarm der Apparatur durch Verbrennen von Zink im trockenen Chlorstrom hergestellt, von wo es direkt in den Anodenraum überdestillierte.

Der Versuch wurde nach 3 Tagen abgebrochen, um eine einwandfreie Messung des Masseneffektes der Wanderungsgeschwindigkeiten für Zink im $\mathrm{ZnCl}_{2} \mathrm{zu}$ erhalten. Hierfür muß bei Versuchsende ein längeres Stück des Diaphragmas noch natürliches Isotopengemisch enthalten, so daß der erfolgte Isotopentrans-

*z. Zt. am Theodor-Kocher-Institut der Universität Bern. port nicht durch stattgehabte Rückdiffusion oder Rückkonvektion der Beobachtung teilweise entgeht. Zur Bestimmung des Masseneffektes aus der stationären Endeinstellung der Apparatur müßte man die effektive Diffusionskonstante kennen, was die Meßgenauigkeit beeinträchtigen würde.

\section{Gestalt und Herstellung des Trenn- rohres}

Die Versuchsanordnung zeigt Abb.1. Der wichtigste Teil des aus Supremax-Glas hergestellten Gefäßes für die Schmelzen ist das Trennrohr (Abb. 2). Dieses ist auf $18 \mathrm{~cm}$ Länge 4,1 mm weit, einschließlich der abschließenden Glasfilter-Platten $21,1 \mathrm{~cm}$ lang, hat $0,9 \mathrm{~mm}$ Wandstärke und ist gefüllt mit Glaspulver von 0,2 bis $0,4 \mathrm{~mm}$ Korngröße, welches als die zwischen zwei Sieben der Maschenweiten DIN 30 und DIN 40 verbleibende Fraktion erhalten worden war. Die Filterplatten (Durchmesser $9 \mathrm{~mm}$, Dicke $4 \mathrm{~mm}$ ) waren aus Glaspulver der Korngröße 0,6 bis $1,0 \mathrm{~mm}$ (DIN 11-14) hergestellt. Das Trennrohr hat an seinen Enden einen erweiterten Querschnitt, damit die Stromdichte an diesen kritischen Stellen vermindert wird und damit die Filterplatten nicht unbequem klein sein müssen. An der unteren Filterplatte wurde darauf geachtet, daß der Rand des Glasrohres nicht nach unten über die Platte hinausragt, weil sonst dort im Betrieb störende Gasblasen hängenbleiben können.

Die Zerkleinerung von Glas zur Gewinnung von Pulver geschah in einem Stahlzylinder mit Kolben unter einer hydraulischen Presse. Das Pulver wurde vor und nach dem Sieben mit konz. Salpetersäure gekocht, gewaschen und getrocknet. Die Filterplatten wurden in einem elektrischen Muffelofen bei $1000^{\circ} \mathrm{C}$ hergestellt, indem jeweils

1 Literatur siehe A. Kle m m, Z. Naturforschg. 6a, 487 [1951]. 


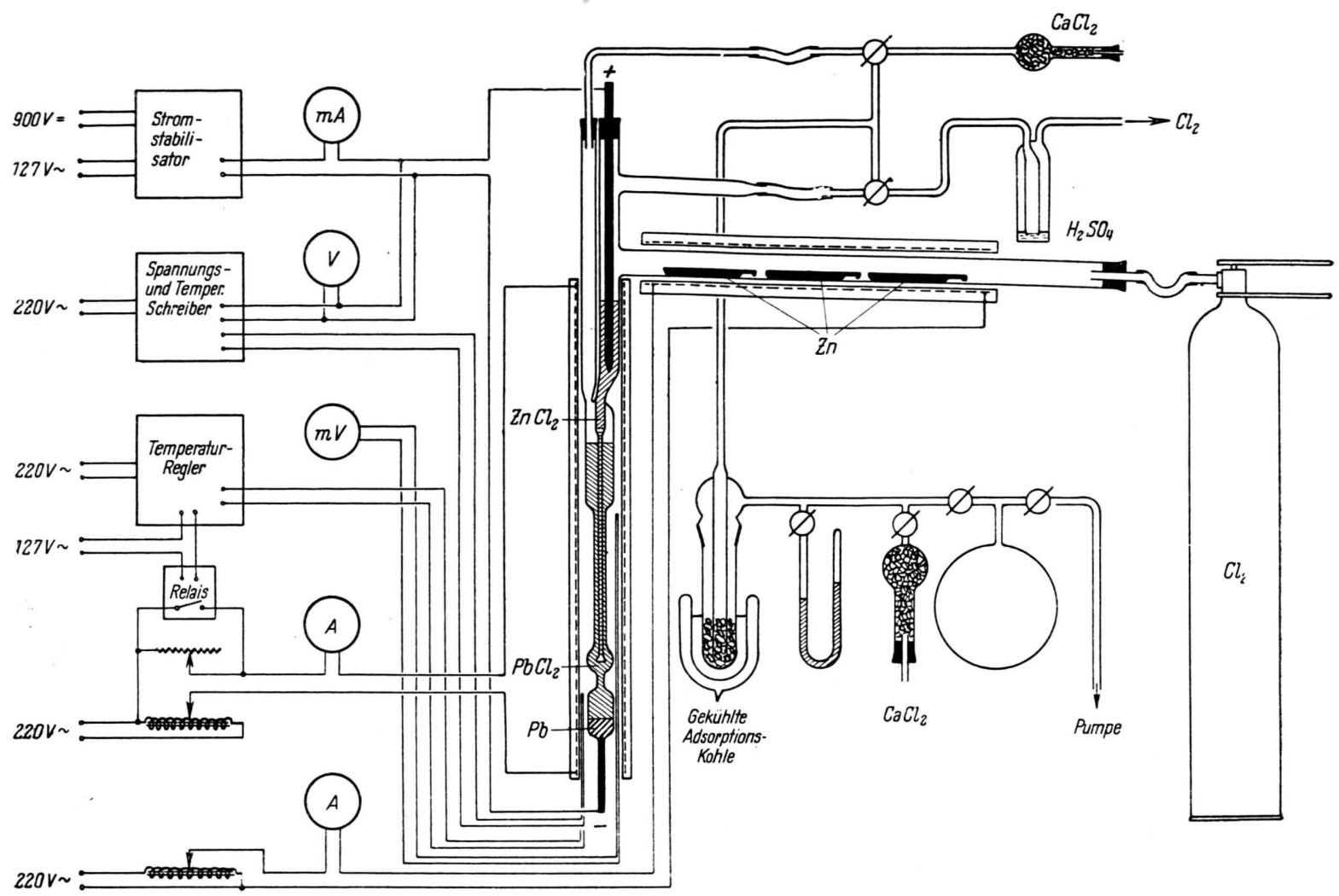

Abb. 1. Elektrolysegefäß und Öfen (maßstäblich) mit Zubehör (schematisch).

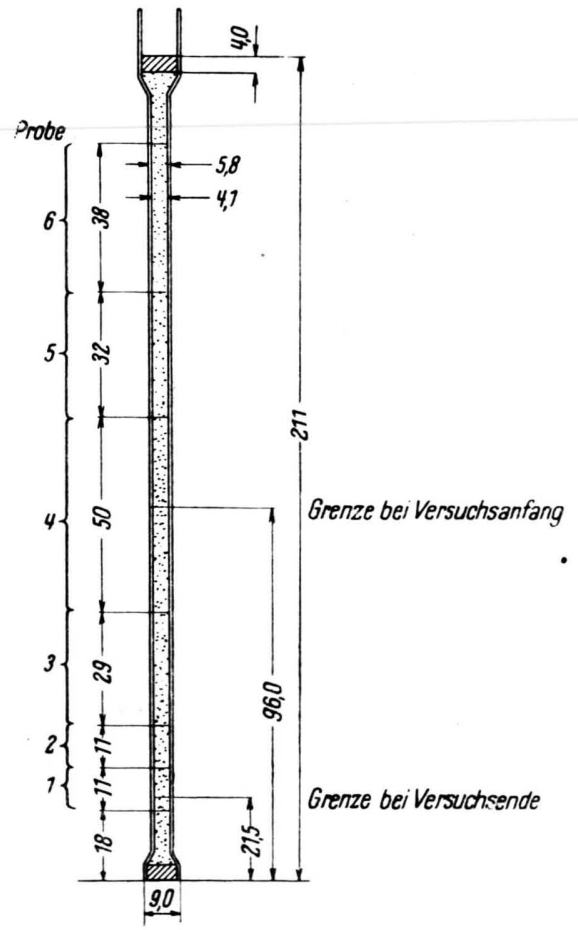

Abb. 2 Das Trennrohr und seine Zerlegung. Längen in mm.
5 mit dem Glaspulver gefüllte zweiteilige Formen aus Elektrodengraphit, die auf eine Kachel aufgereiht und zum Schutz gegen zu schnellen Abbrand mit Porzellantiegeln bedeckt waren, 20 Minuten lang im Ofen belassen wurden. Zur Herstellung des Trennrohres wurde zunächst die obere Filterplatte eingeschmolzen, dann wurde in das auf dem Kopf stehende Rohr heißes Glaspulver portionsweise und abwechselnd mit heftigem Klopfen so dicht als möglich eingefüllt. Wenn man nun sofort die untere Filterplatte einschmolz, dann war nach einigen Tagen oft ein Hohlraum im Glaspulver zu beobachten, weil sich das Glaspulver offenbar langsam gesetzt hatte. Deshalb wurde die untere Filterplatte erst eingeschmolzen, nachdem das Trennrohr einige Tage offen gestanden hatte.

Die Durchlässigkeit der Trennrohre wurde in verschiedenen Herstellungsstadien verfolgt, indem die Zeit gemessen wurde, in der in einem $2-l$-Kolben der Luftdruck von 20 auf $60 \mathrm{~mm} \mathrm{Hg}$ anstieg, wenn der Kolben über das Trennrohr mit der Atmosphäre in Verbindung stand. Beim fertigen Trennrohr betrug diese Zeit 42 sec.

Durch Messung von Durchmesser und Länge verschiedener Trennrohre und Wägung in ungefülltem und gefülltem Zustand wurde bei Kenntnis der Dichte von Supremaxglas $\left(2,438 \mathrm{~g} / \mathrm{cm}^{3}\right)$ festgestellt, daß im Mittel $59 \%$ ces Rohrvolumens mit Pulver erfüllt ist und $41 \% \pm 2 \%$ für die Aufnahme der Schmelze leer bleibt. Diese Zahl hängt von der Homogenität des Pulvers, aber nicht von seiner Feinheit ab. 


\section{Chemikalien}

Das Zink (in Stäben) AsT. war ein B.D.H. Laboratory Reagent der Britis h Drug Houses, London.

Das Bomben-Chlor von E.K.A. (B o h u s) war absolut trocken (durch $\mathrm{H}_{2} \mathrm{SO}_{4}$-Dusche) und enthielt $<0,01 \%$ organischer Halogen-Verbindungen. Die chlorführenden Schläuche waren aus Polyvinylchlorid. Zum Schutz der Pumpe vor aggressiven Gasen war eine mit Adsorptionskohle gefüllte Falle, die mit Aceton-Kohlensäure gekühlt werden konnte, vorgelegt.

Das Bleichlorid, C.B. von C o le man \& B e 11 (USA.) enthielt höchstens $0,005 \%$ Kupfer, 0,1\% Erden und Alkalien, $0,001 \%$ Eisen, 0,000\% Nitrat und 0,01\% Sulfat.

\section{Das F üllen der Apparatur}

Die vom Glasbläser fertiggestellte Apparatur wurde mit Salpetersäure gereinigt, gewaschen und im Trockenschrank bei $140^{\circ} \mathrm{C}$ getrocknet. Dann wurde sie eingebaut, im wagrechten Schenkel mit $40 \mathrm{~g} \mathrm{Zn}$, auf 3 Schiffchen verteilt, beschickt und 12 Stdn. unter dem Vakuum einer rotierenden Ölpumpe belassen, wobei der senkrechte gläserne Ofen auf $500^{\circ} \mathrm{C}$ erhitzt war. Das Bleichlorid, welches in einem großen Reagensglas in einem anderen Ofen unter Vakuum so lange geschmolzen worden war, bis es kein Gas mehr abgab, wurde nach dem Lufteinlassen in die Apparatur mittels eines dünnwandigen Glastrichters durch den linken oberen Stutzen in die Apparatur eingegossen, und zwar bis zur Höhe der oberen Filterplatte. Auf diese Weise erfüllte das Bleichlorid das ganze Diaphragma von unten her und verdrängte dort die Luft, trat jedoch nicht in den Anodenraum ein, wo eine spätere Mischung von $\mathrm{PbCl}_{2}$ mit $\mathrm{ZnCl}_{2}$ vermieden werden sollte. Nachdem die Apparatur nochmals bis zum Aufhören der Gasabgabe des Bleichlorids evakuiert worden war, wurde in der Apparatur wieder Luftdruck hergestellt und dann Chlor eingeleitet, welches über eine mit Schwefelsäure gefüllte Waschflasche, an der seine Strömungsgeschwindigkeit zu beobachten war, ins Freie entweichen konnte. Durch Erhitzen des wagerechten Röhrenofens auf etwa $850^{\circ}$ wurde das Zink entzündet. Es brannte mit hellem weißen Licht, und das gebildete $\mathrm{ZnCl}_{2}$ sammelte sich im Anodenraum an. Die zunächst unscharfe Grenze $\mathrm{PbCl}_{2}-$ $\mathrm{ZnCl}_{2}$ wurcle dabei in das Trennrohr hineingedrückt. Wenige Minuten nach dem Einschalten des Elektrolysenstromes wurde sie völlig scharf und deutlich sichtbar. Der wagerechte Supremaxglas-Schenkel hatte sich infolge der $850^{\circ}$ etwas gebogen.

\section{Die elektrische Einrichtung}

600 Volt der Gleichspannung wurden einem bis 2 A belastbaren Selengleichrichter entnommen und weitere $200 \mathrm{~V}$ von einem Röhrengleichrichter geliefert. Ein Gleichstromstabilisator (Abb. 3) hielt den Elektrolysenstrom auf $230 \mathrm{~mA}$ konstant. Die durch das Nachuntenwandern der Grenze $\mathrm{PbCl}_{2} / \mathrm{ZnCl}_{2}$ systematisch ansteigende Zellenspannung sowie die mit einem Thermoelement in der Höhe cler unteren Filterplatte gemessene Temperatur wurde von einem Papierschreiber registriert. Ein zweites, unmittelbar neben dem ersten angebrachtes Thermoelement steuerte einen

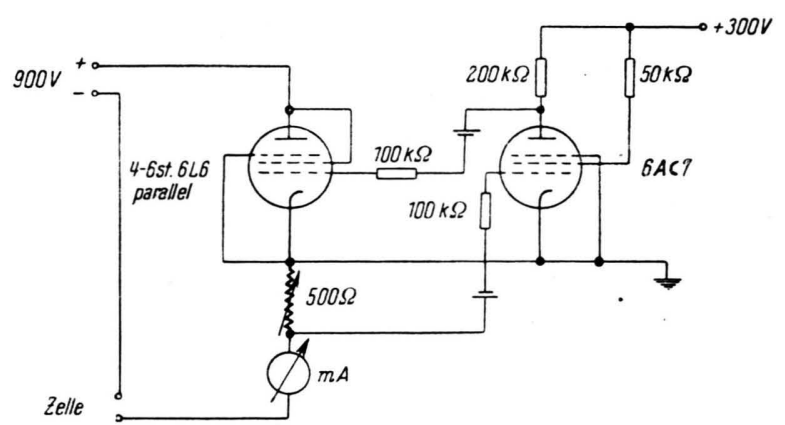

Abb. 3. Schema des Gleichstrom-Reglers.

Temperaturregler, der cie Zeigerbewegung eines Millivoltmeters kapazitiv ausnützt (Whe e l co Instru me n t s Co., Chicago, Modell 221). Durch den Temperaturregler wurde abwechselnd 5 und $6 \mathrm{~A}$ Heizstrom eingeschaltet. Ein drittes, verschiebbares Thermoelement diente zur Messung der räumlichen Temperaturvariation im Ofen.

\section{Verlauf des Versuches}

Während des Versuches war die Temperatur nach Angabe des Temperaturschreibers innerhalb $5^{\circ} \mathrm{C}$ konstant. Sie war so gewählt, daß das Bleichlorid im Kathodenraum unten fest und oben flüssig war, so daß dort die Schmelztemperatur herrschte $\left(501^{\circ} \mathrm{C}\right)$. Dies war vorteilhaft, weil sich dann so wenig als möglich Blei im Bleichlorid löste. Die Einschnürung der Apparatur zwischen der unteren Filterplatte und der Kathode (Abb. 1) trug vollends dazu bei, daß die schwarz gefärbte Bleilösung nur bis zur Höhe dieser Einschnürung anstieg, und daß das Trennrohr durch den klargelben Mantel von Bleichlorid hindurch stets deutlich sichtbar war. Im Ofen neben dem Trennrohr wurde ein Temperaturanstieg nach oben von $520^{\circ} \mathrm{C}$ bis $575^{\circ} \mathrm{C}$ auf $13 \mathrm{~cm}$ Länge gemessen. Die Temperatur im Trennrohr selbst konnte nur indirekt abgeschätzt werden, und zwar aus der Änderung der Zellenspannung bei der Verschiebung der Grenze $\mathrm{PbCl}_{2}-\mathrm{ZnCl}_{2}$. Es gilt nämlich:

$$
\frac{\mathrm{d} V}{\mathrm{~d} x}=i\left(\frac{1}{\varkappa_{1}}-\frac{1}{\varkappa_{2}}\right)
$$

wo $V=$ Zellenspannung, $x=$ Abstand der Grenzfläche von der oberen Filterplatte, $i=$ Stromdichte, $\varkappa_{1}$ und $\varkappa_{2}$ elektrische Leitfähigkeiten von $\mathrm{ZnCl}_{2}$ und $\mathrm{FbCl}_{2}$. Aus den experimentellen Werten $\mathrm{d} V / \mathrm{d} x$ $=12,5 \mathrm{Volt} / \mathrm{cm}$ und $i=4,27 \mathrm{~A} / \mathrm{cm}^{2}$ sowie der bekannten starken Temperaturabhängigkeit der Leitfähigkeiten, besonders des $\mathrm{ZnCl}_{2}$, ergab sich die Temperatur im Trennrohr an der Stelle der Grenze zu $650^{\circ} \mathrm{C}\left(\varkappa_{1}=0,32 \Omega^{-1} \mathrm{~cm}^{-1}, \varkappa_{2}=2,08 \Omega^{-1} \mathrm{~cm}^{-1}\right)$. 
Dies stimmt mit der Beobachtung überein, daß sich einerseits das Trennrohr während des Versuchs etwas verbogen hatte, daß aber andererseits bei der nachträglichen Zerlegung das Glaspulver im Trennrohr noch nicht zusammengesintert war, sondern von selbst herausfiel. Bei einem Vorversuch wurde festgestellt, daß die Zellenspannung bei Erhöhung der Ofentemperatur um $1^{\circ} \mathrm{C}$ um etwa 1 Volt stieg. Infolge des Verbrauchs von $\mathrm{PbCl}_{2}$ im Reserveraum wanderte die Grenzfläche kontinuierlich nach unten, und die

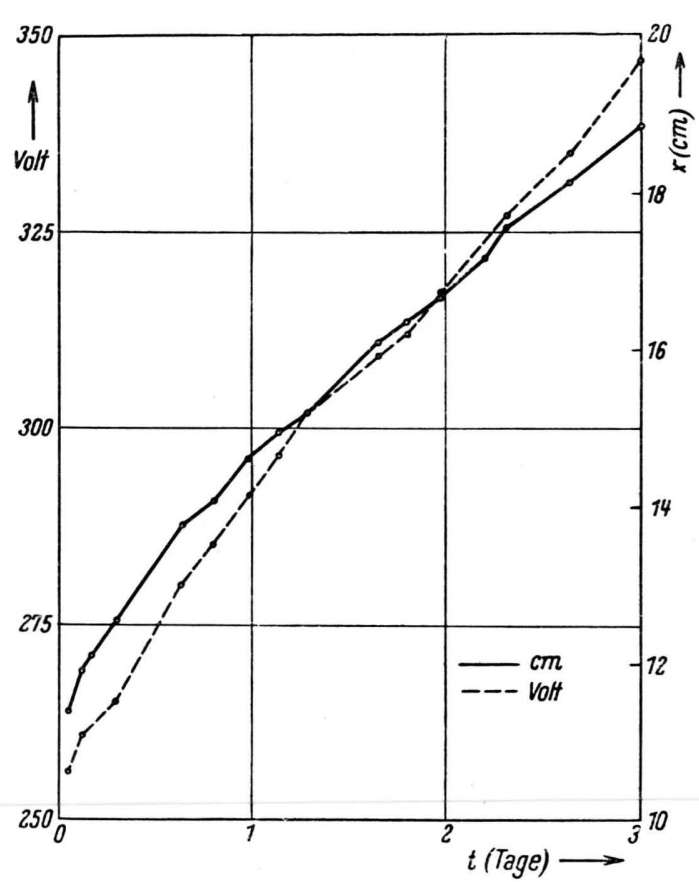

Abb. 4. Zeitlicher Anstieg der Elektrolyse-Spannung infolge der zunehmenden Länge der $\mathrm{ZnCl}_{2}-$ Säule.

Zellenspannung stieg entsprechend, wie es Abb. 4 zeigt. Dabei blieb der Quotient der Niveaudifferenzen zwischen der Grenze $\mathrm{ZnCl}_{2}-\mathrm{PbCl}_{2}$ und den Oberflächen des $\mathrm{ZnCl}_{2}$ und $\mathrm{PbCl}_{2}$ nahezu konstant bei 1,895. Daß er etwas von dem rein hydrostatischen Gleichgewichtswert 2,01 abwich, ist als Folge der Wechselwirkungen zwischen den wandernden Ionen und der Glaswand nicht verwunderlich. Als die Grenze $2 \mathrm{~cm}$ von der unteren Filterplatte entfernt war, wurde der Versuch abgebrochen.

Die Abkühlung wurde durch Entfernung der unteren Asbestisolation so vorgenommen, daß das $\mathrm{PbCl}_{2}$ von unten nach oben fortschreitend erstarrte, damit nicht das später erstarrende $\mathrm{ZnCl}_{2}$ bei der Volumenverminderung des $\mathrm{PbCl}_{2}$ in den Kathodenraum hineingesaugt wurde, sondern rechtzeitig durch Erstarren des $\mathrm{PbCl}_{2}$ im unter- sten Trennrohrstück ein Verschluß gebildet wurde. Nach Absprengung des Glas- und $\mathrm{PbCl}_{2}$-Mantels wurde dann das Trennrohr gemäß Abb. 2 in einzelne Proben zerlegt, die wegen der Hygroskopie des $\mathrm{ZnCl}_{2}$ sofort in Glasröhrchen eingeschmolzen wurden.

\section{Chemische Analyse}

Nachdem die zugeschmolzenen Glasröhrchen mit den Trennrohrstücken gewogen worden waren, wurden sie aufgebrochen und in Wasser gebracht, so daß sich $\mathrm{PbCl}_{2}$ und $\mathrm{ZnCl}_{2}$ auflösten. Das gesamte Glas wurde dann auf Papierfiltern gewaschen und nach Veraschung des Papiers gewogen. Durch Differenzbildung ergab sich die Menge Chlorid in jeder Probe (Tab. 1). Da Probe $1 \mathrm{PbCl}_{2}$ und

\begin{tabular}{|c|c|c|c|c|}
\hline $\begin{array}{c}\text { Probe } \\
\text { Nr. }\end{array}$ & $\begin{array}{c}\text { Länge } \\
(\mathrm{cm})\end{array}$ & $\begin{array}{c}\mathrm{ZnCl}_{2} \\
(\mathrm{mg})\end{array}$ & $\mu f_{n}$ & $f_{n}$ \\
\hline 1 & 0,9 & 124 & $-12,93$ & 166,6 \\
2 & 1,1 & 148 & $-6,16$ & 79,4 \\
3 & 2,9 & 398 & $-1,58$ & 20,4 \\
4 & 5,0 & 690 & - & - \\
5 & 3,2 & 437 & - & - \\
6 & 3,8 & 570 & - & - \\
\hline
\end{tabular}

Tab. 1. Längen, Gewichte und Vervielfältigungsfaktoren der Proben 1-6.

$\mathrm{ZnCl}_{2}$ enthielt, wurde hier das im Filtrat vorhandene Blei als Sulfat gefällt und gewogen. Es ergab sich, daß die Probe $33,9 \mathrm{mg} \mathrm{PbCl}$, enthielt. Außerdem enthielt Probe 1, wie aus einem schwach blauen Schimmer an der Stelle der Grenze $\mathrm{PbCl}_{2}-\mathrm{ZnCl}_{2}$ erkenntlich war, eine Spur Eisen, das sich an der Grenze angereichert hatte. Auf Grund eines Vorversuches, bei dem das Eisen als $\mathrm{Fe}(\mathrm{SCN})_{3}-$ Lösung kolorimetrisch bestimmt worden war, konnten wir abschätzen, daß es sich diesmal um weniger als $1 \mathrm{mg} \mathrm{Fe}$ handelte.

Die $\mathrm{ZnCl}_{2}$-Lösungen wurden zwecks Bildung von Zinkat mit $\mathrm{NaOH}$ versetzt, und das Zink an Anoden aus Pt-Blech elektrolytisch abgeschieden.

\section{Massenspektrometrische Analyse}

Zur massenspektroskopischen Untersuchung erwies sich das auf Platinblech niedergeschlagene metallische Zink als ungeeignet und wurde mit Salzsäure wieder chloriert. Die so erhaltenen $\mathrm{ZnCl}_{2}$ Proben wurden in der Ionenquelle eines $60^{\circ}$ Massenspektrometers aus einem Öfchen verdampft, durch einen Elektronenstrahl ionisiert und die Isotopenhäufigkeiten am $\mathrm{Zn}^{+}$-Spektrum gemessen. $\mathrm{Da}$ ziemlich leicht $\mathrm{ZnCl}_{2}{ }^{++}$-Ionen auftreten, die das $\mathrm{Zn}^{+}$-Spektrum teilweise überlagern, wurde mit einer sehr kleinen Beschleunigungsspannung für die Elektronen (23 Volt) gearbeitet, die beträchtlich unterhalb des Appearance-Potentials der $\mathrm{ZnCl}_{2}{ }^{+}$-Ionen $(\approx 28 \mathrm{~V})$ 


\begin{tabular}{|c|c|c|c|c|c|c|}
\hline$\frac{8}{0}$ & Masse & 64 & 66 & 67 & 68 & 70 \\
\hline 1 & $\begin{array}{c}\text { Meßwert } \\
\text { Ausgeglichen }\end{array}$ & $\begin{array}{l}100 \\
100\end{array}$ & $\begin{array}{l}38,2 \pm 0,5 \\
38,2\end{array}$ & $\begin{array}{l}5,0 \pm 1,0 \\
4,60\end{array}$ & $\begin{array}{l}17,4 \pm 1,0 \\
17,4\end{array}$ & - \\
\hline 2 & $\begin{array}{c}\text { Meßwert } \\
\text { Ausgeglichen }\end{array}$ & $\begin{array}{l}100 \\
100\end{array}$ & $\begin{array}{l}47,1 \pm 0,5 \\
47,1\end{array}$ & $\begin{array}{l}6,5 \pm 1,0 \\
6,28\end{array}$ & $\mid \begin{array}{l}25,3 \pm 1,0 \\
26,2\end{array}$ & - \\
\hline 3 & $\begin{array}{c}\text { Meßwert } \\
\text { Ausgeglichen }\end{array}$ & $\begin{array}{l}100 \\
100\end{array}$ & $\begin{array}{l}54,2 \pm 0,5 \\
54,2\end{array}$ & $\begin{array}{l}8,8 \pm 1,0 \\
7,74\end{array}$ & \begin{tabular}{|l}
$34,5 \pm 1,0$ \\
34,5
\end{tabular} & - \\
\hline 4 & Meßwert & 100 & $58,2 \pm 1,0$ & $8,6 \pm 1,0$ & $38,6 \pm 1,0$ & - \\
\hline 5 & Meßwert & 100 & $57,4 \pm 1,0$ & $9,2 \pm 1,0$ & $38,7 \pm 1,0$ & - \\
\hline 6 & Meßwert & 100 & $57,0 \pm 0,5$ & $8,7 \pm 1,0$ & $39,0 \pm 1,0$ & - \\
\hline \multicolumn{2}{|c|}{ Tabellenwert } & 100 & 56,90 & 8,32 & 38,05 & 1,27 \\
\hline
\end{tabular}

Tab. 2. Gemessene und ausgeglichene Isotopenhäufigkeiten der Proben Nr. $1-6$, bezogen auf ${ }^{64 Z n}$.

lag. Die für die verschiedenen Proben erhaltenen Isotopenhäufigkeiten zeigt Tab. 2. Die angegebenen Fehler kommen hauptsächlich von schwachen Störlinien her, die besonders bei den Massen 67 und 70 immer vorhanden waren. Deshalb wurde das schwache Isotop bei der Masse 70 überhaupt nicht gemessen. Die Massenspektren wurden bei konstanter Beschleunigungsspannung durch Veränderung des Magnetfeldes ausgemessen. Ein Hilfsmagnet wurde in der Ionenquelle nicht benutzt.

\section{A uswertung}

Zunächst wurden die massenspektrometrisch gefundenen Isotopenhäufigkeiten mittels der Beziehung

$$
\ln \frac{{ }^{m, 64} \mathrm{~V}_{n}}{{ }^{m, 64} \mathrm{~V}_{0}}=\mu f_{n} 2 \frac{m-64}{m+64}
$$

ausgeglichen. Dabei ist ${ }^{m,}{ }^{64} V_{n}$ die Häufigkeit des Isotops der Masse $m$ in bezug auf das Isotop 64 in Probe $n . \quad n=0$ bezeichnet das natürliche Gemisch. $\mathrm{Da}$ die Isotope 64 und 66 die häufigsten sind, war ihr Mischungsverhältnis am genauesten gemessen. Es wurde deshalb benutzt, um zunächst für jede Probe $\mu f_{n}$ zu bestimmen. Mittels dieser in Tab. 1 eingetragenen $\mu f_{n}$-Werte wurden die übrigen Mischungsverhältnisse berechnet. Für die natürlichen Häufigkeiten wurden die Tabellenwerte ${ }^{2}$ eingesetzt. Die Meßwerte sowie das Ergebnis des Ausgleiches sind in Tab. 2

$2 \mathrm{M} \mathrm{at} \mathrm{ta} \mathrm{u} \mathrm{c} \mathrm{h-} \mathrm{F} \mathrm{l} \mathrm{a} \mathrm{m} \mathrm{mer} \mathrm{s} \mathrm{f} \mathrm{e} \mathrm{ld,} \mathrm{Isotopenbericht}$ 1949 (Sonderheft der Z. Naturforschg.).

3 A. K l e m m, Z. Naturforschg. 1, 252 [1946]. und Abb. 5 zusammengefaßt. Man erkennt, daß in Probe 1 bis 3 die schweren Isotope in der erwarteten Reihenfolge abgereichert sind. Der größte vorkommende Trennfaktor besteht zwischen den Isotopen 64 und 70 in Probe 1 und beträgt 3,19. Die Proben 4 bis 6 können als von natürlicher Isotopenzusammensetzung gelten.

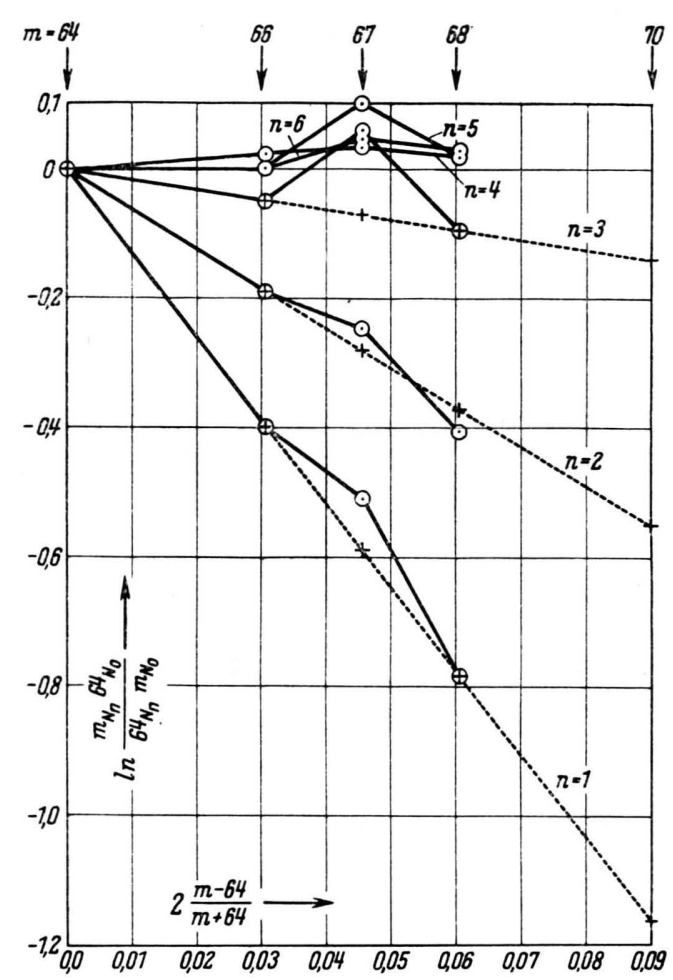

Abb. 5. Abszisse: relative Massendifferenz. Ordinate: Abreicherung der Isotope $66,67,68$ und 70 gegenüber der natürlichen Mischung 0 und dem häufigsten Isotop 64 für die Proben 1 bis 6. Kreise: Meßwerte; Kreuze: Ausgeglichene Werte.

Für das systematische Herausfallen der für die Masse 67 gemessenen Häufigkeitswerte ist die bereits erwähnte Störlinie verantwortlich, die sich wegen der geringen Häufigkeit dieses Isotopes bei der Masse 67 besonders stark auswirkt. Der durch die Gleichung

$$
\mu=\frac{\Delta w}{w} / \frac{\Delta m}{m}
$$

( $w=$ Ionenwanderungsgeschwindigkeit, $m=$ Isotopenmasse) definierte Masseneffekt $\mu$ wurde mittels der Gleichungen ${ }^{3}$

$$
\begin{aligned}
\left(\frac{\Delta w}{w}\right)_{64,66} & =\left(\frac{{ }^{64} N}{{ }^{64} N_{0}}-\frac{{ }^{66} N}{{ }^{66} N_{0}}\right) \frac{F N_{\mathrm{Cl}}}{L}, \\
{ }^{m} N & ={ }^{m} N_{1}+{ }^{m} N_{2}+{ }^{m} N_{3}
\end{aligned}
$$


bestimmt. Dabei ist $F=96500$ Coulomb, $L=0,23 \mathrm{~A}$, 3 Tage $=59600$ Coulomb. $N_{\mathrm{Cl}}$ ist die in den Proben $1-3$ als $\mathrm{ZnCl}_{2}$ enthaltene Menge $\mathrm{Cl}$ in $\mathrm{Mol}$. Da die 3 Proben $670 \mathrm{mg} \mathrm{ZnCl}_{2}$ enthielten, war $\mathrm{N}_{\mathrm{Cl}}$ $=0,00983$.

Es waren nun die absoluten Mengen ${ }^{64,66} \mathrm{~N}$ der Isotope 64 und 66 in den Proben 1 bis 3 zu berechnen. Dies geschah mittels der Gleichung

$$
{ }^{m} N_{n}=N_{n} \frac{{ }^{m, 64} V_{n}}{\sum_{m}^{m, 64} V_{n}},
$$

wobei für die ${ }^{m, 64} V_{n}$ die ausgeglichenen Werte gemäß Tab. 2 eingesetzt wurden. So wurde gefunden

$$
{ }^{64} N N_{0}-\frac{{ }^{66} N}{{ }^{66} N_{0}}=0,150 .
$$

Daraus ergibt sich

$$
\left(\frac{\Delta w}{w}\right)_{64,66}=0,00239 \text { und } \mu=-0,0776
$$

Nach Kenntnis von $\mu$ konnten noch für die Proben 1 bis 3 die Vervielfachungen $f_{n}$ aus den bekannten $\mu f_{n}$-Werten bestimmt werden (Tab. 1). Da sich die Anreicherung auf ungefähr $4 \mathrm{~cm}$ Länge erstreckte, setzen wir $\sqrt{\pi D^{\prime} t}=4 \mathrm{~cm}$ und erhalten mit $t=3$ Tage für die effektive Diffusionskonstante der Zinkionen den Wert $D^{\prime}=1,7 \mathrm{~cm}^{2} /$ Tag.

Dieser im Vergleich zu früheren Versuchen kleine Wert dürfte auf eine verminderte Konvektion im Diaphragma infolge der thermisch günstigeren Lagerung des Trennrohres in einem Bad aus geschmolzenem Bleichlorid zurückzuführen sein.

Die Schmelzelektrolyse wurde in Göteborg am Institut für Physik der Technischen Hochschule durchgeführt, wo der eine von uns (A. K.) als Gast weilte. Herr Prof. Dr. N. R y d e hat die Arbeit durch Schaffung der Voraussetzungen auf das freundlichste unterstützt, Herr fil. kand. $\mathrm{K}$ o n g s tad hat den Röhrengleichrichter und den Stromstabilisator unter Mitwirkung von Herrn Ingenieur Brune entworfen und gebaut, Herr Civiling. R. A n derss on und Herr stud. phys. A. Lodding haben bei den Versuchen selbst geholfen. Herr Dr. R. Lindner vom Institut für Silikatforschung in Göteborg hat uns einige Proben chemisch analysiert. Die Isotopenhäufigkeitsmessungen hat der zweite von uns (H. H.) mit dem Massenspektrometer des Max-Planck-Instituts für Chemie als Gast des Theodor-Kocher-Instituts der Universität Bern unter Mitwirkung von Herrn C. L a n g durchgeführt. Wir möchten allen Beteiligten unseren herzlichsten Dank abstatten.

\title{
Positive Kugelkorona und Schwingungsmessungen
}

\begin{abstract}
Von Wilhelm Fucks und Walter Budde
Aus dem Physikalischen Institut der Rheinisch-Westfälischen Technischen Hochschule Aachen

(Z. Naturforschg. 6 a, 494-504 [1951]; eingegangen am 28. Mai 1951)

Die Koronaentladung läßt sich zu Schwingungsmessungen verwenden. Es werden zunächst statisch ermittelte Kurzschluß- und Arbeitskennwerte der Koronameßstrecke mitgeteilt. Sodann wird die Koronameßstrecke mit schwingender Kathode betrachtet. Mehrere für die Theorie nützliche Begriffe lassen sich in Analogie zur Theorie der Verstärkerröhre definieren. Es zeigt sich, daß für tiefere Frequenzen die statisch ermittelten Kennlinien für instationäre Vorgänge zugrundegelegt werden dürfen. Die Schwingungsform einer quadratischen Glasplatte wird mit der Koronameßmethode bestimmt.
\end{abstract}

$\mathrm{I}^{\mathrm{n}}$ n mehreren vorangegangenen Arbeiten ${ }^{1,2,3}$ wurde von $\mathrm{F} \mathrm{u} \mathrm{c} \mathrm{k} \mathrm{s} \mathrm{gezeigt,} \mathrm{daß} \mathrm{sich} \mathrm{Vorstromentladungen}$ zu trägheits- und rückwirkungsfreien anemometrischen, thermometrischen und mechanischen Schwan-

1 W. Fu cks, Dtsch. Luftfahrtforschg., Untersuch. Mitt. 1202 (31) 2203 [1944]; ebenda 1203 (31) 2203 [1944]; ebenda 1205 (31) 2803 [1944]; ebenda 1230 (31) 0305 [1944]; ebenda 1299 (35) 0308 [1944]; ebenda 1431 (40) 1101 [1944]; FEPI 1943; DVL-Bericht 1943; W. Fucks u. F. Kettel, Dtsch. Luftfahrtforschg., Untersuch. Mitt. 1452 (35) 1801 [1945]; ebenda 1470 [1945]; W. Fucks u. G. Schumacher, ebenda 1189 (35) 1103 [1944]. kungs- und Schwingungsmessungen verwenden lassen. Im folgenden wird über die Grundlagen der Messung von mechanischen Schwingungen mit der Koronaentladung berichtet. Der Entladung zwischen einer Kugel als Anode und einer Platte als Kathode (Abb. 1) kommt hierbei besondere Bedeutung zu, weil bei Messungen an schwingenden Metallfächen diese direkt als Kathode verwendet werden können. Bei

2 W. F u ck s, Z. Naturforschg. 5 a, 89 [1950].

3 W. F u ck s, Z. angew. Physik (im Druck). 